09

\title{
Генерация гармоник в экспериментах с лазерами на свободных электронах в рентгеновском диапазоне - теоретический анализ
}

\author{
(C) К.В. Жуковский \\ Московский государственный университет им. М.В. Ломоносова, физический факультет, кафедра теоретической \\ физики, \\ 119991 Москва, Россия \\ e-mail: zhukovsk@physics.msu.ru
}

Поступило в Редакцию 28 августа 2020 г.

В окончательной редакции 16 сентября 2020 г.

Принято к публикации 22 сентября 2020 г.

Дано аналитическое описание генерации гармоник ондуляторного излучения в лазерах на свободных электронах в различных экспериментах в рентгеновском диапазоне. Выражения для формы линий спектра и интенсивности записаны явно в терминах обобщенных функций Бесселя и Эйри с учетом разброса энергии электронов, размера пучка, эмиттанса, расщепления линий спектра, постоянных компонент магнитного поля. Представленная теория хорошо объясняет спектр и интенсивность излучения гармоник во всех экспериментах на установках ЛСЭ SACLA, LCLS, PAL-XFEL, SwissFEL и др., в различных условиях с длинами волн в широком диапазоне, включая жесткое рентгеновское излучение.

Ключевые слова: ондулятор, лазер на свободных электронах, гармоники, поле, пучки, внеосевые эффекты.

DOI: 10.21883/JTF.2021.03.50529.243-20

\section{Введение}

Развитие теории и эксперименты в области синхротронного (СИ) и ондуляторного (ОИ) излучений привели к появлению источников последнего поколения - лазеров на свободных электронах (ЛСЭ), в которых когерентное излучение генерируется электронами, сгруппированными в микробанчи на расстоянии длины волны излучения друг от друга. Пучки электронов высоких энергий с релятивистским фактором $\gamma=E / m c^{2} \approx 10^{3}-10^{4} \gg 1$, где $m-$ масса электрона, $c$ - скорость света, $E-$ энергия, позволяют получать рентгеновское излучение и исследовать процессы на наномасштабе [1-4]. Для этого нужны качественные пучки с малым разбросом энергии, эмиттансом и отклонением от оси; перечисленные факторы приводят к потерям и ухудшению группировки электронов, особенно значимым для источников рентгеновского излучения. Ввиду сложности решения всего комплекса уравнений, описывающих движение электронов и их взаимодействие с излучением в ЛСЭ, моделирование ЛСЭ обычно проводится численно с применением специальных программ подготовленным персоналом. Нами разработан универсальный аналитический формализм [5-13], использующий обобщенные функции типа Бесселя и Эйри в сочетании с аналитической моделью однопроходного ЛСЭ, которая феноменологически учитывает все основные потери в реальных установках. Это позволяет изучить и проанализировать генерацию гармоник практически в любом ЛСЭ на обычном персональном компьютере без применения численных программ. Новизна исследования заключается в аналитическом учете всех характеристик пучка и ондулятора, учете повышенной чувствительности электрон-фотонного взаимодействия в ЛСЭ на длинах волн высших гармоник к потерям [14], аналитическом описании режима выхода на насыщение в конце ЛСЭ и осцилляций мощности насыщения, а также более реалистичном учете вклада шума электронного банча в начале ЛСЭ. Это дает реалистичное описание спектра и мощности гармоник излучения по всей длине ЛСЭ, включая режим насыщения, и хорошо согласуется с экспериментами в различных условиях с излучением в диапазоне от видимого до рентгеновского.

Магнитное поле в ондуляторе обычно близко к синусоидальному. Однако идеальное синусоидальное магнитное поле не удовлетворяет уравнениям Максвелла во всем пространстве зазора ондулятора, и в реальных устройствах всегда присутствуют высшие гармонические составляющие поля. Кроме того, в ондуляторах с постоянными магнитами иногда специально создают бигармоническое магнитное поле с помощью шиммирования ферромагнитными материалами [15-21]. Гармоники поля позволяют получить усиленное по сравнению с обычным излучение высших гармоник ОИ $[20,22]$. Амплитуда гармоник поля может доходить до 20-30\% основного поля ондулятора $[19,23,24]$. Влияние гармоник поля на излучение реальных электромагнитных ондуляторов изучалось, например, в $[25,26]$, а также в ондуляторах с постоянными магнитами и с электромагнитами в [27-30]. В этих исследованиях был сделан вывод о том, что гармоники поля ондулятора с соответствующей фазой и амплитудой могут усилить излучение гармоник ОИ и дать более высокий коэффициент усиления высших гармоник ЛСЭ. Это подтверждается в недавней работе [31]. В эллиптических ондуляторах также присутствуют гармоники поля, например, в предложенных 
ондуляторах с изменяемой эллиптичностью излучения APPLE-III для SwissFEL [32], Delta [33] для LCLS [34] и др. Численное моделирование излучения эллиптических ондуляторов проводилось, например, в [35].

Ниже мы приведем аналитические формулы, позволяющие точно описать и исследовать влияние гармоник поля на излучение в эллиптических и плоских ондуляторах с гармониками поля произвольной амплитуды. Используя их в аналитической модели однопроходного ЛСЭ (см., например, [9-14]), мы исследуем спектральные характеристики некоторых основных ЛСЭ экспериментов в рентгеновском диапазоне: SACLA, LCLS, PAL-XFEL, SwissFEL, и сравним результаты для мощностей гармоник, их эволюции по длине ондуляторов и ширины спектральных линий с имеющимися экспериментальными данными.

\section{1. Теория ОИ обобщенного эллиптического ондулятора с учетом потерь}

Рассмотрим излучение обобщенного эллиптического ондулятора с учетом непериодических магнитных компонент $H_{x}=H_{0} \rho, H_{y}=H_{0} \kappa$ и гармоник поля с амплитудами $d, d_{1}, d_{2}$ и периодами с кратностью $l, h$, $p$ основному периоду $\lambda_{u, x} \equiv \lambda_{u}$ :

$$
\begin{array}{r}
\mathbf{H}=H_{0}\left(\sin \left(k_{\lambda} z\right)+d \sin \left(p k_{\lambda} z\right)+\rho,\right. \\
\left.d_{1} \sin \left(h k_{\lambda} z\right)+d_{2} \cos \left(l k_{\lambda} z\right)+\kappa, 0\right),
\end{array}
$$

$k_{\lambda}=2 \pi / \lambda_{u, x}, h, l, p \in$ integers, $d, d_{1}, d_{2} \in$ reals.

Конфигурация поля (1) обобщает плоский и эллиптический ондуляторы [7,9]. Следуя по аналогии [36], вычисляем интеграл излучения

$$
\frac{d^{2} I}{d \omega d \Omega}=\frac{\left|\frac{1}{c} \omega \frac{e}{2 \pi} \int_{-\infty}^{\infty} d t[\mathbf{n} \times[\mathbf{n} \times \boldsymbol{\beta}]] \exp [i \omega(t-\mathbf{n r} / c)]\right|^{2}}{c}
$$

где $\mathbf{n} \cong\left(\theta \cos \varphi, \theta \sin \varphi, 1-\theta^{2}\right)-$ единичный вектор в направлении от заряда к наблюдателю, $\theta-$ угол отклонения от оси ондулятора, $\varphi$ - угол вокруг оси ондулятора, $\omega-$ частота излучения, $\boldsymbol{\beta}-$ скорость заряда и $\mathbf{r}$ - его радиус-вектор. В результате получаем интенсивность ОИ в следующем виде:

$$
\begin{aligned}
& \frac{d^{2} I}{d \omega d \Omega} \cong \frac{e^{2} N^{2} \gamma^{2} k^{2}}{c\left(1+k^{2} / 2\right)^{2}} \sum_{n=-\infty}^{\infty} n^{2}\left\{\left|S\left(f_{n}^{1}+f_{n}^{2}\right)\right|^{2}\right. \\
& \left.+\left|\left(\partial S / \partial v_{n}\right) f_{n}^{3}\right|^{2}\right\},
\end{aligned}
$$

где

$$
S \equiv\left(v_{n}, \eta, \beta\right)=\int_{0}^{1} d \tau \exp \left(i\left(v_{n} \tau+\eta \tau^{2}+\beta \tau^{3}\right)\right)
$$

- специальная функция типа Эйри, зависящая от параметра расстройки $v_{n}$, угла отклонения от оси ондулятора $\theta$, угла поворота вокруг оси $\varphi$ и эффективного угла изгиба $\theta_{H}=\frac{2 \pi k}{\sqrt{3}} \frac{k}{\gamma} N\left(\kappa^{2}+\rho^{2}\right)^{1 / 2} \quad$ [36-39], индуцированного непериодическими магнитными компонентами поля $H_{x}=H_{0} \rho$ и $H_{y}=H_{0} \kappa ; \beta \cong 2 \pi n N \frac{\left(\gamma \theta_{H}\right)^{2}}{1+k^{2} / 2}$ и $\eta=\frac{4 \pi^{2} N^{2} n k \gamma \theta}{\left(1+k^{2} / 2\right)}(\kappa \cos \varphi-\rho \sin \varphi)-$ аргументы функции $S, f_{n}^{1,2,3}$ - коэффициенты Бесселя:

$$
\begin{gathered}
f_{n, x}^{1}=\frac{d_{1}}{h}\left(J_{n+h}^{n}+J_{n-h}^{n}\right)+i \frac{d_{2}}{l}\left(J_{n+l}^{n}-J_{n-l}^{n}\right), \\
f_{n, x}^{2}=\frac{2}{k} \gamma \theta \cos \varphi J_{n}^{n}, \\
f_{n, y}^{1}=\left(J_{n+1}^{n}+J_{n-1}^{n}\right)+\frac{d}{p}\left(J_{n+p}^{n}+J_{n-p}^{n}\right), \\
f_{n, y}^{2}=\frac{2}{k} \gamma \theta \sin \varphi J_{n}^{n}, \\
f_{n}^{3}=2 \sqrt{3} \gamma \theta_{H} J_{n}^{n} / k,
\end{gathered}
$$

которые зависят от обобщенных функций Бесселя $J_{n}^{m}$; последние задаются в виде громоздких интегралов тригонометрических функций $[37,38]$. В реальных устройствах амплитуда гармоник поля относительно мала, $d$, $d_{1}, d_{2} \lesssim 0.3$, и тогда $J_{n}^{m}$ приближенно записывается в следующем виде:

$$
\begin{aligned}
& J_{n}^{m} \approx \int_{-\pi}^{\pi} \frac{d \alpha}{2 \pi} \exp \left[i \left(n \alpha+\theta \cos \varphi \frac{\lambda_{u} m k}{\lambda_{n} n \gamma} \sin \alpha\right.\right. \\
& \left.\left.+\frac{\lambda_{u} m k^{2}}{8 \lambda_{n} n \gamma^{2}} \sin (2 \alpha)\right)\right],
\end{aligned}
$$

где $k=H_{0} \lambda_{u, x} e / 2 \pi m_{e} c^{2} \cong \lambda_{u}[\mathrm{~cm}] H_{0}[k G] / 10.7-$ ондуляторный параметр, $\varphi$ - азимутальный угол, $\theta-$ полярный угол от оси ондулятора, $\lambda_{n}-$ резонансы ОИ:

$$
\begin{gathered}
\lambda_{n}=\frac{\lambda_{u}}{2 n \gamma^{2}}\left(1+\frac{k^{2}}{2} \Delta+(\gamma \Theta)^{2}\right), \\
\Delta=1+\left(\frac{d}{p}\right)^{2}+\left(\frac{d_{1}}{h}\right)^{2}+\left(\frac{d_{2}}{l}\right)^{2}, \\
\Theta^{2}=\theta^{2}+\theta_{H}^{2}-\sqrt{3} \theta_{H} \theta \frac{\rho \sin \varphi-\kappa \cos \varphi}{\sqrt{\kappa^{2}+\rho^{2}}} .
\end{gathered}
$$

Обсуждение свойств обобщенной функции типа Эйри $S \mathrm{~S}$ и ее производной $\partial S / \partial v_{n}$, которые описывают 

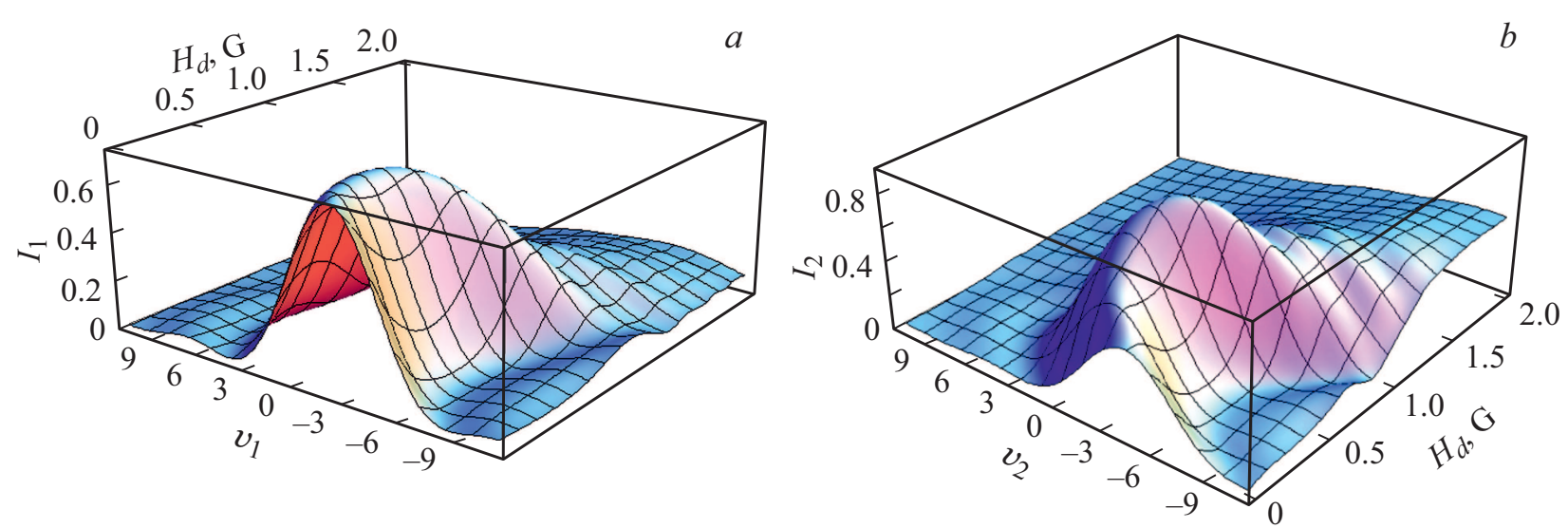

Рис. 1. Линии спектра излучения гармоник $n=1(a)$ и $2(b)$ ондулятора PAL-XFEL в зависимости от непериодического поля $H_{d}$ для ОИ под углом $\gamma \theta=0.067$.

форму линий спектра ОИ в присутствии слабого непериодического магнитного поля в ондуляторе, проводилось ранее, например, в [40]. В чисто периодическом поле $H_{d}=0$, и получаем функцию sinc: $S\left(v_{n}, 0,0\right)=$ $=\exp \left(i v_{n} / 2\right) \operatorname{sinc}\left(v_{n} / 2\right)$, как в обычной теории ОИ.

Используя формулы (3)-(9), исследуем влияние компонент поля $H_{x}=H_{0} \rho$ и $H_{y}=H_{0} \kappa$ на линии спектра гармоник $n=1$ и 2 ондулятора PAL-XFEL [41] длиной $L=5 \mathrm{~m} \quad$ с периодом $\lambda_{u}=2.57 \mathrm{~cm} \quad$ и параметром $k=1.87$. Интенсивность гармоник $I_{n}\left(v_{n}, H_{d}\right)=\sqrt{\left|S\left(f_{n}^{1}+f_{n}^{2}\right)\right|^{2}+\left|\left(\partial S / \partial v_{n}\right) f_{n}^{3}\right|^{2}}$ под углом расходимости $\gamma \theta=0.067$ к оси показана на рис. 1; видно, что постоянное поле может вызвать заметную вторую гармонику ОИ $(b)$ и отчасти компенсировать эффект влияния угла $\theta$ на нечетные гармоники $(a)$. Для этого достаточно напряженности $\sim 0.5 \mathrm{G}$, порядка поля Земли.

Описанный выше эффект накапливается по длине секции ондулятора и может быть заметным в длинных ондуляторах; это было отмечено, в частности, при строительстве ЛСЭ LCLS [42, 43] с ондуляторами длиной $3.5 \mathrm{~m}$. Фокусирующие и дефокусирующие элементы производят корректировку пучка на оси между секциями ондулятора. Естественно, для корректного расчета ОИ нами учитывается разброс энергий электронов с помощью следующей свертки в выражении для интенсивности ОИ: $\int_{-\infty}^{\infty} \frac{d^{2} I\left(v_{n}+4 \pi n N \varepsilon, \theta\right)}{d \omega d \Omega \sqrt{2 \pi} \sigma_{e}} \exp \left(-\frac{\varepsilon^{2}}{2 \sigma_{e}^{2}}\right) d \varepsilon ;$ эмиттанс и реальные размеры пучка также учитываются аналитически.

Важную роль в формировании спектра ОИ и ЛСЭ играют бетатронные колебания [44,45], хорошо изученные еще на ранних этапах развития теории ОИ [46-50]. Они вызывают расщепление линий спектра на гармоники, отстоящие друг от друга на бетатронную частоту $\omega_{\beta}=\sqrt{2} \pi c k \delta / \lambda_{n} n \gamma$, где $\delta=\sqrt{1+d^{2}+d_{1}^{2}+d_{2}^{2}}$ учитывает влияние высших гармоник поля (1); $\delta=1$ для обычного плоского ондулятора. Случай плоского бигармонического ондулятора подробно рассмотрен в [25]; наши результаты в пределе $d_{1}=d_{2}=0$ сводятся к соответствующим выражениям в [25]. Для релятивистских электронов $\gamma \gg 1$ и бетатронное расщепление происходит на малую частоту $\omega_{\beta} \propto \omega_{n} / \gamma$. Расщепление гармоники $n$ на бетатронные гармоники $p$ описывается в терминах коэффициентов Бесселя $f_{n}$ рядом по $p$ :

$$
\begin{gathered}
f_{n}^{1,2} \rightarrow \sum_{p} \tilde{J}_{p}(\xi, \xi) f_{n}^{1,2}, \\
\tilde{J}_{p}(\xi, \xi)=\frac{1}{2 \pi} \int_{0}^{2 \pi} \cos (p q-\xi \sin q-\xi \sin 2 q) d q,
\end{gathered}
$$

где функции Бесселя $\tilde{J}_{p}(\xi, \xi)$ зависят от удаления $y_{0}$ электрона от оси пучка:

$$
\begin{gathered}
\xi=\frac{\pi^{2} y_{0}^{2} k \delta}{2 n \gamma \sqrt{2} \lambda_{u} \lambda_{n}}=\frac{\pi^{2} \gamma y_{0}^{2} k \delta}{\sqrt{2} \lambda_{u}^{2}\left(1+\left(k^{2} / 2\right)\right)}, \\
\xi=\frac{2 \pi \theta y_{0}}{n \lambda_{n}}=\frac{4 \pi \theta y_{0} \gamma^{2}}{\lambda_{u}\left(1+\left(k^{2} / 2\right)\right)} .
\end{gathered}
$$

Бетатронные колебания приводят к появлению четных гармоник на оси ондулятора с коэффициентами Бесселя

$$
f_{n}^{4} \cong \sum_{p} \frac{\sqrt{2} \pi y_{0} \delta}{\lambda_{u}}\left(\tilde{J}_{p+1}(\xi, \xi)-\tilde{J}_{p-1}(\xi, \xi)\right) J_{n}^{n}(\xi),
$$

где $n-$ номер гармоники ОИ, $p-$ номер бетатронной гармоники и $J_{n}^{n}\left(\xi_{i}\right)-$ функция Бесселя (7). Иерархия величин коэффициентов Бесселя такова: максимальный вклад дают гармоники $n=1,3,5: f_{n=1,3}^{1} \sim 0.3-0.8$ и $f_{n=5}^{1} \sim 0.1-0.3$ в зависимости от ондулятора; все угловые вклады обычно существенно меньше: $f_{n}^{2}<0.1$; непериодическое поле обычно компенсируется и $f_{n}^{3}<f_{n}^{2}$; бетатронные колебания в релятивистских пучках дают еще меньший вклад: $f_{n}^{4} \sim 10^{-3}-10^{-2}$. Отметим, что при этом бетатронное расщепление линии спектра гармоники $n$ в рентгеновских ЛСЭ может происходить на много 

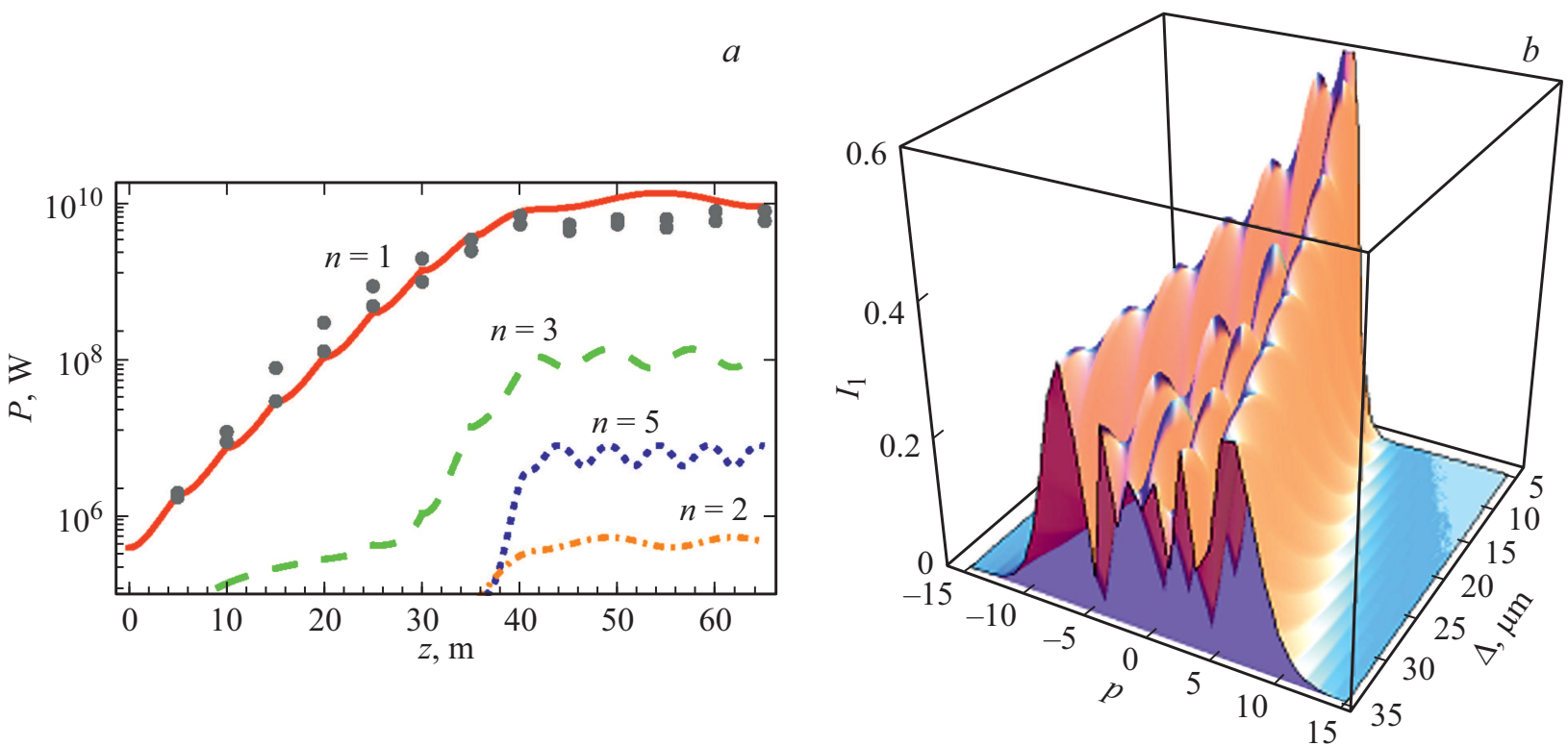

Рис. 2. $a$ - эволюция мощности гармоник ЛСЭ в эксперименте PAL-XFEL с $E=8 \mathrm{GeV}, \lambda_{1}=0.144 \mathrm{~nm}, \sigma_{e}=0.18 \cdot 10^{-4}$, $I_{0}=2.5 \mathrm{kA}$. Экспериментальные величины измерений показаны точками. $b-$ расщепление линии основного тона в зависимости от расстояния от оси.

бетатронных гармоник $p=-10 \ldots 10$, соответствующее уширение существенно и должно учитываться.

Для моделирования излучения ЛСЭ в разд. 2 используем аналитическую модель однопроходных ЛСЭ в ее последнем варианте [37,38].

\section{2. Моделирование излучения некоторых рентгеновских ЛСЭ}

В эксперименте PAL-XFEL с ондуляторами длиной $5 \mathrm{~m}$, периодом $\lambda_{u}=2.6 \mathrm{~cm}, k=1.87$ и излучением на длине волны $\lambda=0.144 \mathrm{~nm}[41]$, происходит довольно сильное расщепление линий на $\sim 15-20$ бетатронных гармоник $p$ (рис. $2, b$ ). Несмотря на это, полная ширина линии основного тона оказывается мала, $\Delta \lambda \sim 0.14 \mathrm{pm}$, спектральная плотность линии такова: $\Delta \lambda / \lambda \approx 1.0 \cdot 10^{-3}$. Для сравнения в ЛСЭ LCLS [43] на той же длине волны $\lambda=0.15 \mathrm{~nm}$ расщепление почти на порядок меньше и $\Delta \lambda / \lambda<0.1 \cdot 10^{-3}$ благодаря значительно лучшему качеству пучка. Рост мощности излучения в эксперименте PAL-XFEL с $\lambda_{1}=0.144 \mathrm{~nm}$ показан на рис. 2, $a$.

Мы получили неплохое согласие с экспериментом в режиме экспоненциального роста; интересно, что мы получили мощность насыщения немного больше, чем в эксперименте, в то время как численная симуляция авторов эксперимента дает мощность меньше, чем измеренная. Смоделированная эволюция мощности излучения гармоник по длине ондуляторов представлена на pис. 2, $a$; в режиме насыщения третья гармоника составляет $<1 \%$; излучение пятой гармоники, по-видимому, будет очень слабым из-за дифракционного предела; вторая гармоника тоже слабая, несмотря на выраженное бе- татронное расщепления линий спектра. Гармоники ЛСЭ PAL-XFEL не измерялись экспериментально; их излучение затруднено, так как слабое неравенство $\varepsilon_{x, y} \lesssim \lambda_{n} / 4 \pi$ не выполнено уже для основного тона: $\varepsilon=3.5 \times 10^{-11}$ и $\lambda_{1} / 4 \pi=1.1 \times 10^{-11}$, не говоря о гармониках.

B LCLS-экспериментах [43,51] с излучением волн длиной $\lambda_{1}=1.5$ и $0.15 \mathrm{~nm}$ ЛСЭ состоит из 33 секций длиной $3.4 \mathrm{~m}$ каждая, ондуляторы имеют период $\lambda_{u}=3 \mathrm{~cm}$, $k=H_{0} \lambda_{u} e / 2 \pi m c^{2}=3.5$, ток $I_{0}=1-3 \mathrm{kA}$, энергия электронов $E=4.3-13.6 \mathrm{GeV}$. В эксперименте в жестком рентгеновском диапазоне измерялась первая и третья гармоника излучения и моделирование этого эксперимента не приводится для краткости. Мы проанализировали эксперимент с мягким рентгеновским излучением на длине волны $\lambda_{1}=1.5 \mathrm{~nm}$ от электронов с энергией $E=4.3 \mathrm{GeV}$ и током $I_{0}=1 \mathrm{kA}[51]$, где зарегистрированы вклады второй гармоники $P_{2} \approx 0.04-0.1 \%$, третьей гармоники $P_{3} \approx 2-2.5 \%$, а также оценен вклад пятой гармоники $P_{5} \approx 0.2-0.25 \%$. Результаты аналитического моделирования мощности и спектра представлены на рис. 3 ; использованы данные установки и эксперимента из [51]. Мы получили длину усиления $L_{g} \approx 1.5 \mathrm{~m}$, насыщение после $L_{s} \approx 25 \mathrm{~m}$, и мощности гармоник, включая вторую, соответствующие данным измерений (рис. 3, $a$ ). Бетатронные колебания дают малый вклад. Для $n=2$ имеем $f_{2, y}^{4} \sim 2 \cdot 10^{-3}$, что значительно меньше вклада расходимости: $f_{2 ; y} \sim 0.02$. По данным [43-51] траектории пучка с энергией $E_{e}=4.3 \mathrm{GeV}$ уходят с оси на $10-20 \mu \mathrm{m}$ на длине усиления $L_{\text {gain }} \approx 1.5 \mathrm{~m}$. Это отклонение сравнимо с размером фотонного и электронного пучков: $\sigma_{\gamma} \approx 16 \mu \mathrm{m}, \sigma_{e} \approx 22 \mu \mathrm{m}$. Возникает эффективный угол $\theta_{\gamma} \approx 0.07$, который добавляет мощности второй 


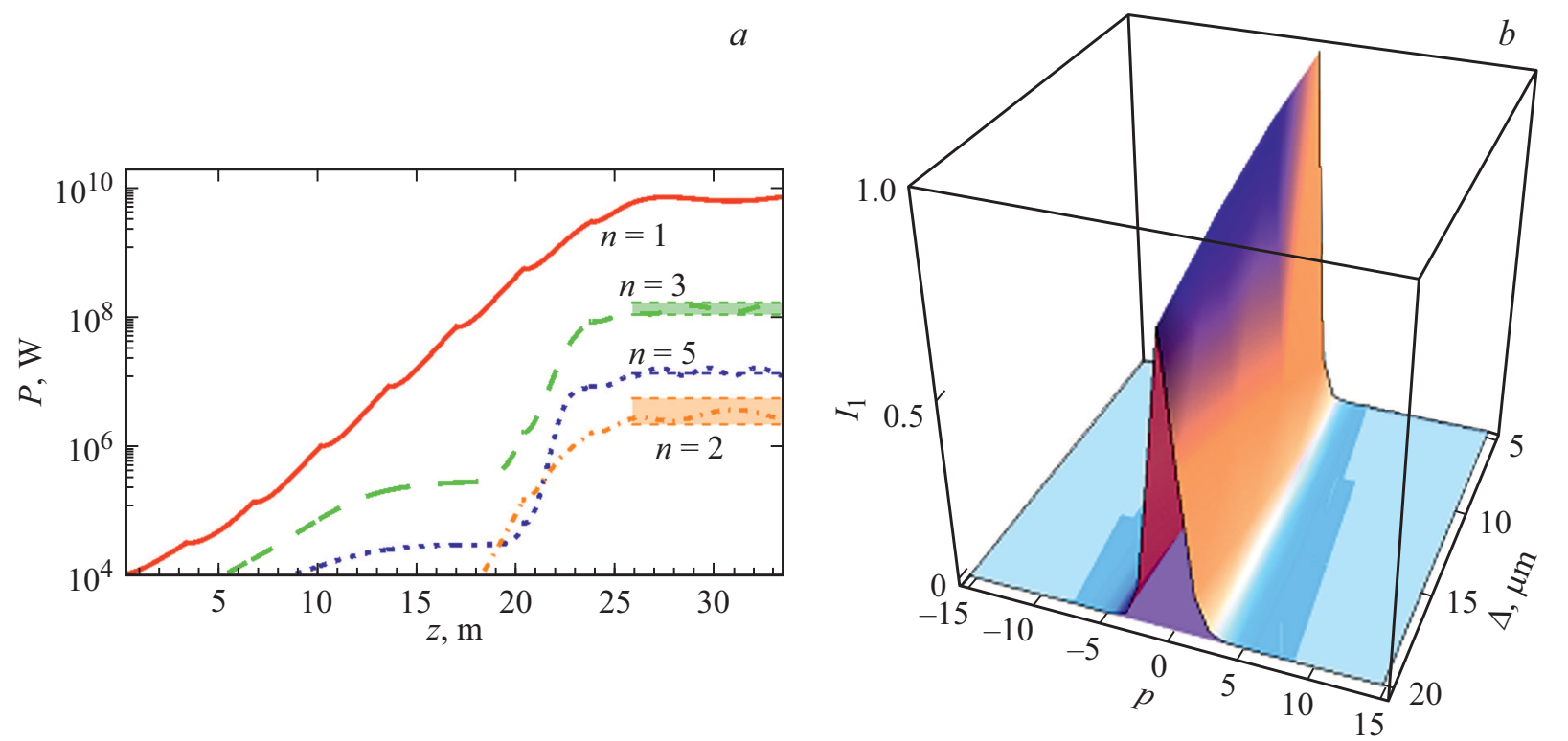

Рис. 3. $a$ - эволюция мощности гармоник ЛСЭ в эксперименте LCLS с $E=4.3 \mathrm{GeV}, \lambda_{1}=1.5 \mathrm{~nm}, \sigma_{e}=0.3 \cdot 10^{-4}$, $I_{0}=1 \mathrm{kA}$. Экспериментально допустимые диапазоны измеренных величин обозначены цветными зонами справа: $P_{3} \approx(2-2.5 \%) P_{1}$, $P_{2} \approx(0.04-0.1 \%) P_{1}$, оценка для $P_{5} \sim 0.1 P_{3} . b-$ расщепление линии основного тона в зависимости от расстояния от оси.

гармоники и немного ослабляет третью и пятую гармоники; с учетом этого теоретическая мощность гармоник (рис. 3) согласуется с экспериментом.

Бетатронное расщепление линий мало (рис. $3, b$ ) и соответствует $p \approx-2 \ldots+2$. С учетом пяти гармоник, получаем относительную ширину $\Delta \lambda / \lambda \sim 1.5 \cdot 10^{-3}$, сравнимую с теоретической шириной линии в режиме экспоненциального усиления, которая, в свою очередь, соответствует параметру Пирса: $\delta \lambda / \lambda \approx \sqrt{\rho \lambda_{u} / L_{s}} \approx$ $\approx \rho \approx 0.0012$. Эти значения хорошо соотносятся с расчетной шириной спектра для LCLS, которая, согласно разработчикам, составляет $\sim 0.1 \%$ [43]; экспериментальные значения оказались несколько выше: 0.2-1\% [43].

В эксперименте SACLA для мягкого рентгеновского излучения [52] ЛСЭ состоит из 3 секций и 777 периодов $\lambda_{u}=1.8 \mathrm{~cm}$; генерировалось когерентное излучение в диапазоне $1-12 \mathrm{~nm}$. Мы рассмотрели эксперимент с излучением на длине волны основного тона $\lambda=12 \mathrm{~nm}$ мощностью $0.22 \mathrm{GW}$ (энергией $E=110 \mu \mathrm{J}$ ), в котором измеренная доля третьей гармоники составляет $\sim 0.3 \%$. При этом использовался ондулятор с параметром дипольности $k=2.1$, пучок электронов с энергией $E=780 \mathrm{MeV}$ и током $I=300$ А [52]. Мы получили теоретическую мощность гармоник $P_{1} \approx 0.2 \mathrm{GW}$, $P_{3} \approx 0.5 \%$, длины усиления и насыщения $L_{g}=1 \mathrm{~m}$ и $L_{s} \approx 12 \mathrm{~m}$, в согласии с экспериментом (рис. $4, a$ ); теоретическая мощность второй гармоники мала, экспериментальные данные о ней отсутствуют.

Расщепление линии ОИ также мало (рис. 4, $b$ ); учитывая $\gamma \approx 1500$ и расщепление на три гармоники, имеем ширину $\Delta \lambda / \lambda \sim 2 \cdot 10^{-3}$, сравнимую с теоретической шириной линии в режиме экспоненциального усиления: $\delta \lambda / \lambda \approx \sqrt{\rho \lambda_{u} / L_{s}} \approx 0.15 \%$, которая почти точно равна параметру Пирса $\rho \approx 0.0015$ и соответствует заявленному в эксперименте.

В другом недавнем ЛСЭ эксперименте SACLA использовались электроны высокой энергии $E=7.8 \mathrm{GeV}$ и короткий банч длительностью $10 \mathrm{fs}$ с малым разбросом энергии $\sigma_{e}=0.0925 \%$, который нес заряд $270 \mathrm{pC}$. Сильнейший ток $27 \mathrm{kA}$ создавал в ондуляторах мощный фотонный импульс с энергией $0.78 \mathrm{~mJ}$ на длине волны излучения $\lambda \approx 0.125 \mathrm{~nm}$ (энергия фотонов $9.85 \mathrm{keV}$ ) [53], a насыщение достигалось уже после $35 \mathrm{~m}$ длины ондуляторов. Это почти в два раза короче, чем длина насыщения в экспериментах LCLS и PAL-XFEL. Мы смоделировали и проанализировали этот эксперимент с самоусилением спонтанного излучения (SASE FEL) на установке SACLA. Результаты аналитического моделирования мощности гармоник излучения и значения для основного тона по данным [53] приведены на рис. 5. В отсутствии исчерпывающей информации о параметрах пучка в [53] мы использовали данные из [54,55], дополняющие [53]: период ондулятора $\lambda_{u}=1.8 \mathrm{~cm}$ [54], параметр дипольности $k \approx 2.1$.

Теоретический расчет дает длину насыщения $L_{s} \approx 32 \mathrm{~m}$, длину усиления $L_{g} \approx 2.05 \mathrm{~m}$ и мощность насыщения $\sim 13 \mathrm{GW}$, длительность фотонного импульса $\sim 6.3 \mathrm{fs}$; эти результаты полностью соответствуют экспериментальным значениям в [53]. Уровень мощности, соответствующий энергии фотонного импульса в эксперименте $\sim 0.780 \mathrm{~mJ}$, обозначен штриховой линией на рис. 5,a. Высшие гармоники не регистрировались; условие $\varepsilon \lesssim \lambda / 4 \pi$ не вполне удовлетворяется даже для основного тона, не говоря о гармониках. По сравнению с предыдущими экспериментами SACLA [56] насыщение в рассматриваемом эксперименте наступает значительно 


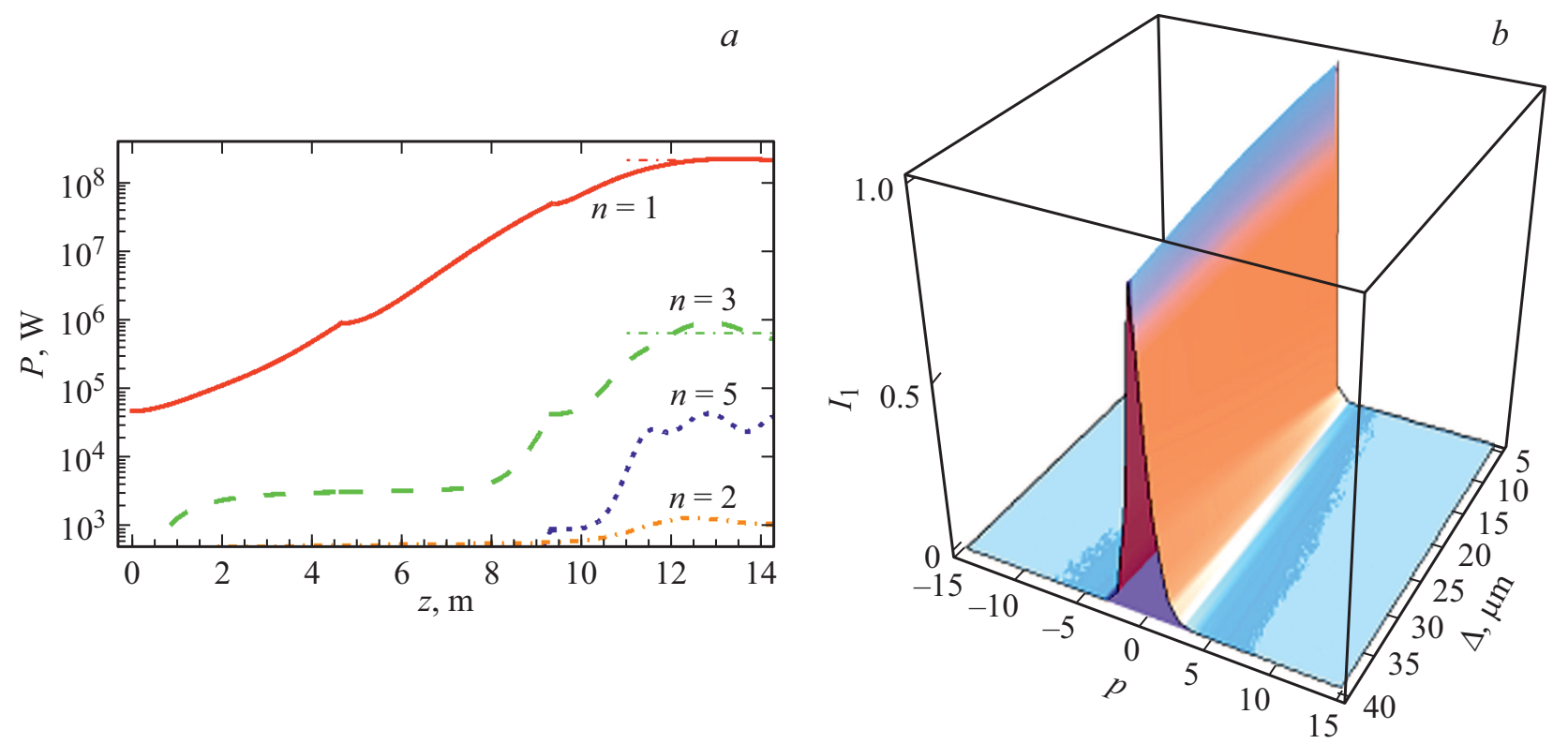

Рис. 4. $a-$ эволюция мощности гармоник ЛСЭ в эксперименте SACLA на длине волны $\lambda_{1}=12.4 \mathrm{~nm} \mathrm{c}$ энергией $E=780 \mathrm{MeV}$, $\sigma_{e}=1.3 \cdot 10^{-3}, I_{0}=300$ А. Экспериментальные значения мощности насыщения обозначены тонкими линиями справа; $b-$ расщепление линии основного тона в зависимости от расстояния от оси.
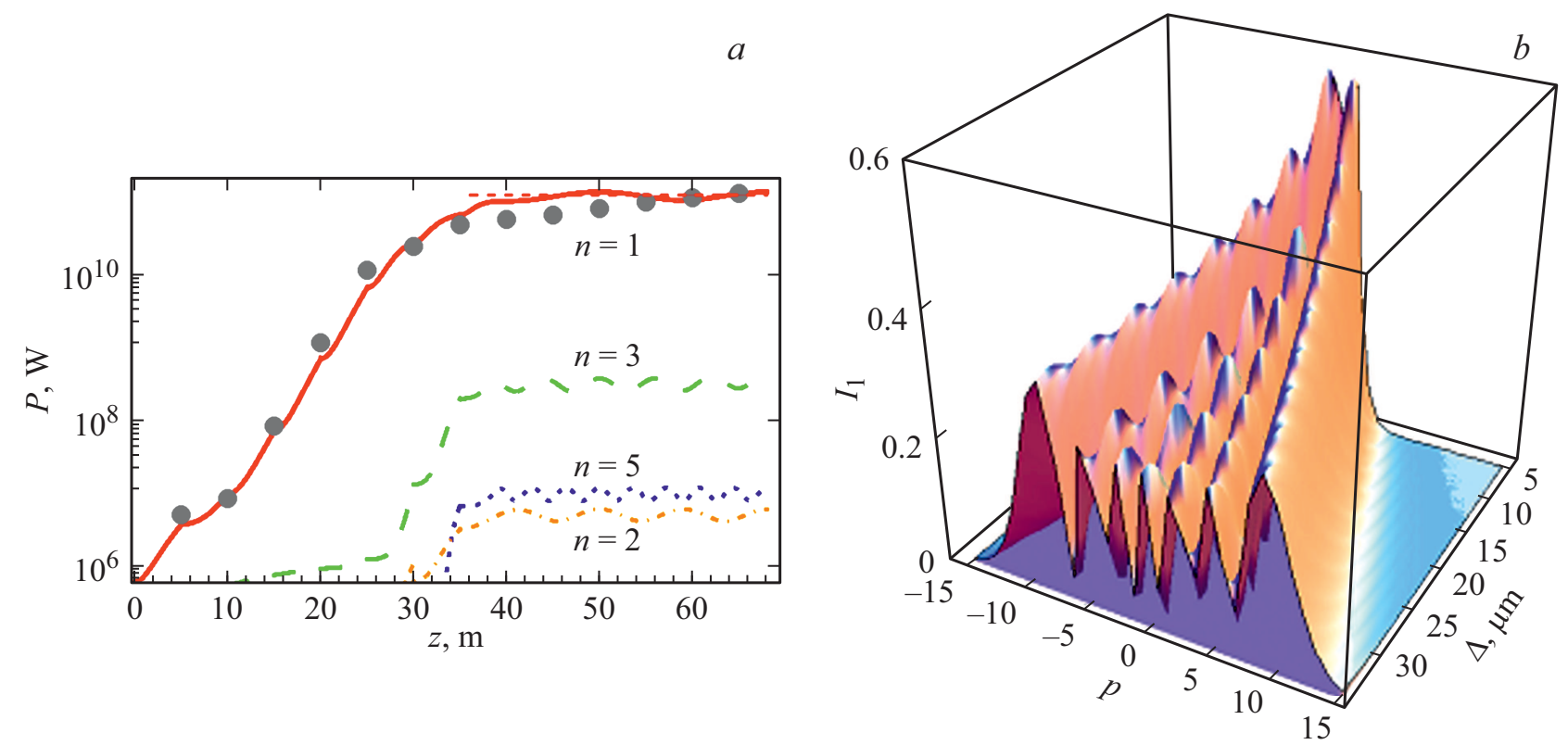

Рис. 5. $a-$ эволюция мощности гармоник ЛСЭ в сильноточном эксперименте SACLA на длине волны $\lambda_{1}=0.125 \mathrm{~nm}$ с энергией $E=7.8 \mathrm{GeV}, \sigma_{e}=0.0925 \%, I_{0}=27 \mathrm{kA}$. Значение мощности фотонного импульса, соответствующее измеренной энергии $0.78 \mathrm{~mJ}$, обозначено штриховой линией справа, экспериментальные значения мощности обозначены серыми точками; $b-$ расщепление линии основного тона в зависимости от расстояния $\Delta$ от оси.

раньше и его мощность больше за счет огромного тока $I=27 \mathrm{kA}$. В то же время расщепление линии спектра тоже значительно больше, чем в ранних экспериментах [57,58]: в последнем эксперименте [53] мы насчитываем $\sim 20$ гармоник в расщепленной линии основного тона (рис. $5, b$ ), что дает теоретическое значение спектральной плотности $\Delta \lambda / \lambda \sim 0.25 \%$, близкое к экспери- ментальному значению $\approx 0.5 \%$ для излучения ЛСЭ в режиме SASE [53]. В то же время значение параметра Пирса $\rho_{1}=0.0008$ и величина $\delta \lambda / \lambda \approx \sqrt{\rho \lambda_{u} / L_{s}} \approx 0.0007$ оказываются почти на порядок меньше, чем измеренная спектральная плотность $0.5 \%$. Таким образом, оценка, полученная нами на основе расщепления линий, ближе к экспериментальному значению. 


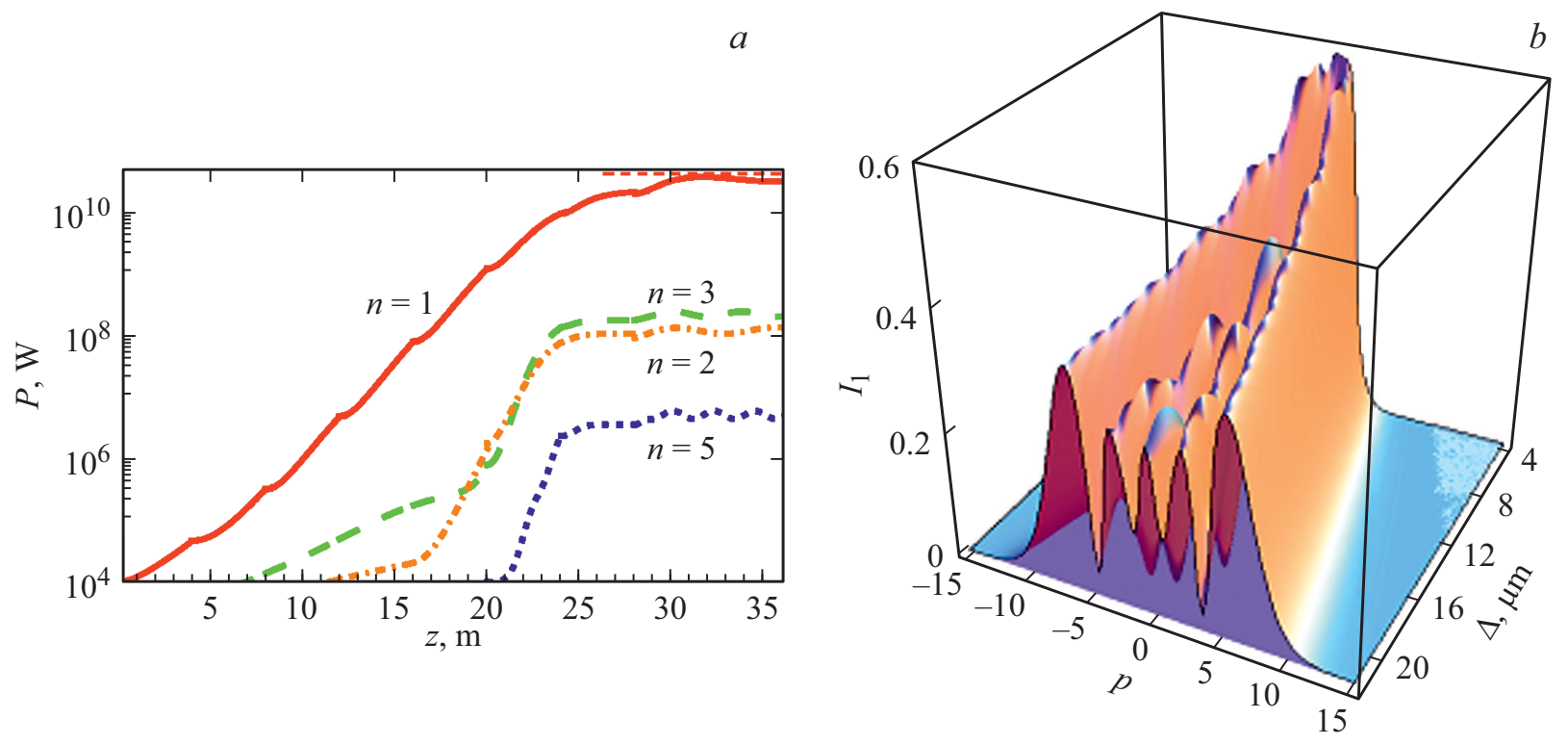

Рис. 6. $a$ - эволюция мощности гармоник ЛСЭ в эксперименте SwissFEL на длине волны $\lambda_{1}=0.1 \mathrm{~nm}$ с энергией $E=5.8 \mathrm{GeV}$, $\sigma_{e}=0.006 \%, I_{0}=5 \mathrm{kA}$. Мощность фотонного импульса, соответствующая измеренной энергии $1 \mathrm{~mJ}$, обозначена красной штриховой линией (в online версии); $b$ - расщепление линии основного тона в зависимости от расстояния $\Delta$ от оси.

В заключении рассмотрим ЛСЭ эксперименты на установке SwissFEL по генерации жесткого рентгеновского излучения в диапазоне $\sim 1.5-0.1 \mathrm{~nm}$ на ондуляторной линии Aramis [59-62]. Особенностью установки является малый разброс энергии, $350 \mathrm{keV}$, что означает $\sigma_{e}=0.006 \%$ для энергии $E=5.8 \mathrm{GeV}$ электронов, которые излучают на длине волны $\lambda_{1}=0.1 \mathrm{~nm}$. Другие данные эксперимента таковы: заряд электронного банча равен $0.2 \mathrm{nC}$, энергия фотонного импульса $E_{\gamma}=1 \mathrm{~mJ}$, его длительность $\tau_{\gamma}=24 \mathrm{fs}$. Исходя их этого, оценка мощности излучения составила $\sim 40 \mathrm{GW}$, длительность электронного импульса $\tau_{e}=40 \mathrm{fs}$, электронный ток $5 \mathrm{kA}$. Смоделированная нами эволюция мощности излучения по длине ондуляторов представлена на рис. 6, $a$. Полученная нами теоретическая мощность насыщения $\approx 40 \mathrm{GW}$ отлично соответствует энергии $E_{\gamma}=1 \mathrm{~mJ}$ импульса длительностью $\tau_{\gamma}=24 \mathrm{fs}$. Это видно на рис. $6, a$, где сплошная линия соответствует мощности гармоники с $n=1$, и штриховая линия справа вверху соответствует мощности насыщения фотонного импульса с энергией $E_{\gamma}=1 \mathrm{~mJ}$ длительностью $\tau_{\gamma}=24 \mathrm{fs}$. Насыщение наступает на длине $L_{s} \sim 25 \mathrm{~m}$, длина усиления $L_{g} \sim 1.4 \mathrm{~m}$.

Несмотря на малую величину дипольного параметра ондулятора $k=1.2$, получаем заметную третью гармонику ЛСЭ с мощностью $P_{3} \approx 0.25 \mathrm{GW}$. Ее вклад составляет $\sim 0.7 \%$; это значение близко к теоретически максимальному возможному для данного ондулятора, и оно реализуется благодаря малому разбросу энергии электронов. Вторая гармоника излучения возникает изза конечного угла электрон-фотонного взаимодействия $\theta=\sigma_{\text {photon }} / L_{\text {gain }} \sim 6 \mu \mathrm{rad}$ в фотонном пучке сечением $\sigma_{\text {photon }} \approx \sqrt{\sigma_{x, y} \sqrt{\lambda_{1} L_{g} / 4 \pi}} \sim 9 \mu$ m, и электронном пучке сечением $\sigma_{x, y}=\sqrt{\varepsilon_{x, y} \beta_{x, y}} \sim 22 \mu \mathrm{m}$, где $\beta_{x, y}$ - параметры Твисса, $\varepsilon_{x, y}$ - эмиттансы. Теоретическая мощность второй гармоники $\sim 0.4 \%$ (см. рис. $6, a$ ). Бетатронные колебания дают пренебрежимо малый вклад в генерацию второй гармоники по сравнению с угловыми эффектами.

Отметим, что в отличие от SwissFEL, в ЛСЭ PALXFEL пучок с энергией $E=7.8 \mathrm{GeV}$ имел значительно больший разброс энергий $-\sigma_{e}=0.018 \%$ [41]. Если бы разброс энергии электронов в ЛСЭ SwissFEL был такой же, как в PAL-XFEL, т.е. $\sigma_{e}=0.018 \%$, то мощности высших гармоник в SwissFEL были бы почти на порядок меньше и длины усиления и насыщения были бы заметно больше. Именно благодаря высокомоноэнергетическому пучку электронов в SwissFEL излучение имеет максимально возможную мощность на минимально возможной длине насыщения.

Расщепление линии спектра SwissFEL получено нами аналитически и представлено на рис. $6, b$. Вычисленная теоретическая ширина линии ЛСЭ SwissFEL с учетом расщепления составляет $\sim 0.1 \%$, что полностью соответствует заявленным значениям $0.05-0.15 \%$ [60].

В заключение отметим, что нами проведено также моделирование ЛСЭ экспериментов LEUTL $[63,64]$ в видимом и UV-A-диапазонах, а также других экспериментов ЛСЭ. Во всех случаях результаты теоретического анализа спектра и мощности соответствуют экспериментам, что говорит о правильности учета всех основных факторов, влияющих на излучение ЛСЭ; моделирование ЛСЭ European XFEL [65] не проводилось изза недостатка доступных данных по динамике излучения в ондуляторах. 


\section{3. Результаты и выводы}

Проведенное теоретическое исследование генерации гармоник ЛСЭ точно аналитически учитывает влияние всех параметров на излучение ЛСЭ и его спектральные характеристики в реальных условиях. Нами смоделирована и изучена генерация гармоник в рентгеновском диапазоне в экспериментах ЛСЭ LCLS, SACLA, PAL-XFEL и SwissFEL в рентгеновском диапазоне. Гармоники поля ондулятора и бетатронные колебания не могут вызывать мощную 2-ю гармонику излучения.

В эксперименте LCLS энергия электронов $E=$ $=13.6 \mathrm{GeV}$ больше, чем во всех других рассмотренных рентгеновских ЛСЭ. Малый разброс энергии и высокое качество пучка, а также значение ондуляторного параметра $k=3.5$, обеспечивают заметное присутствие гармоник излучения. Расщепление линий спектра мало по сравнению с ЛСЭ PAL-XFEL, где излучается та же длина волны. Отклонение траекторий электронов от оси на длине усиления ЛСЭ составляет в некоторых секциях $\sim 15 \mu \mathrm{m}$, что сравнимо с размером пучка. Это вызывает заметную, до $0.1 \%, 2$-ю гармонику; 3я гармоника достигает $2.5 \%$, что объясняется очень хорошим пучком и большим параметром дипольности $k=3.5$, пятая гармоника составляет $10 \%$ от третьей, что полностью согласуется с оценкой авторов эксперимента. Вычисленная нами спектральная плотность, $\Delta \lambda / \lambda \sim 0.15 \%$ согласуется с расчетным значением для установки LCLS, однако ее экспериментальные значения несколько выше: 0.2-0.7\%.

ЛСЭ PAL-XFEL имеет близкие к LCLS характеристики и длины волн излучения, но худшее качества пучка. Это приводит к заметному расщеплению линий спектра в PAL-XFEL: линия $\lambda_{1}=1.5 \mathrm{~nm}$ на $\sim 9$ гармоник, $\Delta \lambda \sim 1.7 \mathrm{pm}$, и линия $\lambda_{1}=0.15 \mathrm{~nm}$ на $\sim 15-18$ бетатронных гармоник, $\Delta \lambda \sim 0.2 \mathrm{pm}$; рассчитанная спектральная ширина линии: $\Delta \lambda / \lambda \sim 1.5 \times 10^{-3}$. Гармоники излучения не регистрировались. Разброс энергий электронов пучка PAL-XFEL больше, чем в эксперименте LCLS, и это препятствует излучению высших гармоник, так как электрон-фотонное взаимодействие на их длинах волн более чувствительно к потерям. Эволюция основного тона, смоделированная нами аналитически, длина усиления и другие характеристики полностью соответствует эксперименту.

В эксперименте SACLA в мягком рентгеновском диапазоне высокое качество пучка дает слабую вторую гармонику; мощность основного тона $P_{1} \approx 0.2 \mathrm{GW}$, третья гармоника составляет $\sim 0.5 \%$. Расщепление линии спектра мало и дает спектральную плотность $\Delta \lambda / \lambda \sim 2 \cdot 10^{-3}$, сравнимую с теоретической шириной линии: $\delta \lambda / \lambda \approx \sqrt{\rho \lambda_{u} / L_{s}} \approx 0.15 \%$, которая совпадает с полученным значением параметра Пирса $\rho \approx 0.0015$; эти данные также соответствуют эксперименту. В эксперименте с сильноточным ЛСЭ с током $27 \mathrm{kA}$ генерируется излучение на длине волны $0.125 \mathrm{~nm}$. Насыщение происходит на длине $L_{s} \approx 32 \mathrm{~m}$, т.е. значительно раньше, чем в ЛСЭ LCLS и PAL-XFEL с подобной длиной волны; длина усиления $L_{g} \approx 2.05 \mathrm{~m}$, динамика мощности излучения и мощность насыщения $\sim 13 \mathrm{GW}$ также соответствуют измеренным в эксперименте. Отсутствие высших гармоник в этом эксперименте, как и в эксперименте PAL-XFEL, по-видимому, объясняется тем, что условие $\varepsilon \lesssim \lambda / 4 \pi$ на эмиттанс не вполне удовлетворяется даже для основного тона, не говоря о гармониках. Расщепление линии излучения основного тона в этом эксперименте происходит на $\sim 20$ бетатронных гармоник, что значительно больше, чем в ранних экспериментах SACLA [56,57]. Полученное нами теоретическое значение спектральной плотности $\Delta \lambda / \lambda \sim 0.0025$ близко к заявленному значению $\sim 0.5 \%$ для излучения ЛСЭ в режиме SASE [53]. Параметр Пирса $\rho_{1}=0.0008$ и величина $\delta \lambda / \lambda \approx \sqrt{\rho \lambda_{u} / L_{s}} \approx 0.0007$ оказываются почти на порядок меньше, чем измеренная спектральная плотность $0.5 \%$.

В эксперименте SwissFEL высокое качество моноэнергетического пучка электронов с разбросом энергии $\sigma_{e}=0.006 \%$ обеспечивает максимальные характеристики при минимальных потерях. Излучается длина волны $0.1 \mathrm{~nm}$; рассчитанная теоретическая мощность насыщения $\sim 40 \mathrm{GW}$ соответствует заявленной энергии фотонного импульса $E_{\gamma}=1 \mathrm{~mJ}$ длительностью $\tau_{\gamma}=24 \mathrm{fs}$. Длина насыщения $L_{s} \sim 25 \mathrm{~m}$, длина усиления $L_{g} \sim 1.4 \mathrm{~m}$. Из-за очень малого разброса энергий электронов заметно излучение гармоник ЛСЭ в ондуляторе с $k=1.2$ : третья гармоника составляет $\sim 0.7 \%$ и вторая гармоника $\sim 0.4 \%$. Рассчитанная нами теоретическая спектральная плотность $\sim 0.1 \%$ соответствует экспериментальным значениям $0.05-0.15 \%$.

Проведенное теоретическое исследование генерации гармоник в ЛСЭ показывает хорошее согласие полученных нами спектральных характеристик с измеренными величинами для всех исследованных рентгеновских и других ЛСЭ. Это подтверждает обоснованность подхода и аналитических методов исследования, которые позволяют рассчитать спектральные свойства практически любого ЛСЭ, не прибегая к разработке и использованию сложных численных программ для моделирования ЛСЭ, и не требуют привлечения соответствующих программных специалистов. Аналитические методы исследования позволяют выявить причины генерации гармоник и объяснить спектральные свойства излучения в каждом случае, а также проанализировать влияние каждого параметра установки на излучение ЛСЭ.

\section{Конфликт интересов}

Автор заявляет, что у него нет конфликта интересов.

\section{Список литературы}

[1] B.W.J. McNeil, N.R. Thompson. Nature Photonics, 4, 814 (2010).

[2] C. Pellegrini, A. Marinelli, S. Reiche. Rev. Mod. Phys., 88, 015006 (2016). 
[3] G. Margaritondo. Rivista del Nuovo Cimento, 40 (9), 411 (2017).

[4] V.G. Bagrov, G.S. Bisnovaty-Kogan, V.A. Bordovitsyn, A.V. Borisov, O.F. Dorofeev, V.Ya. Epp, Y.L. Pivovarov, O.V. Shorokhov, V.C. Zhukovsky. Synchrotron Radiation Theory and Its Development, ed. V.A. Bordovitsyn (Word Scientific, Singapore, 1999), p 447.

[5] K. Zhukovsky. J. Phys. D, 50, 505601 (2017).

[6] K. Zhukovsky. J. Optics, 2018. 20 (9), 095003 (2018).

[7] K. Zhukovsky. Results in Physics, 13, 102248 (2019).

[8] K. Zhukovsky, A. Kalitenko. J. Synchrotron Rad., 26, 159 (2019).

[9] K. Zhukovsky. J. Synchrotron Rad., 26, 1481 (2019).

[10] К.В. Жуковский, А.М. Калитенко. Изв. вузов. Физика, 62 (2), 153 (2019).

[11] К.В. Жуковский. Изв. вузов. Физика, 62 (6), 109 (2019).

[12] К.В. Жуковский. ЖТФ, 89 (3), 426 (2019).

[13] К.В. Жуковский. ЖТФ, 90 (8), 12 (2020).

[14] К.В. Жуковский. Вестн. Моск. ун-та. Физ. Астрон., 5, 60 (2019).

[15] N. Nakao, M. Kokubo, K. Imasaki, M. Fujita, K. Ohkubo, A. Moon, P.K. Roy, H. Tanaka, N. Ohigashi, Y. Sunawaki, K. Mima, S. Nakai, C. Yamanaka. Nucl. Instr. Meth. Phys. Res. A, 407, 374 (1998).

[16] G. Sharma, G. Mishra, M. Gehlot. J. Instrum., 9, T01002 (2014).

[17] F. Bazouband, B. Maraghechi. J. Plasma Phys., 81 (3), 905810305 (2015).

[18] M. Asakawa, K. Mima, S. Nakai, K. masaki, C. Yamanaka. Nucl. Instr. Meth. Phys. Res. A, 318, 538 (1992).

[19] M. Asakawa, N. Inoue, K. Mima, S. Nakai, K. Imasaki, M. Fujita, J. Chen, C. Yamanaka, N. Nakao, T. Agari, T. Asakuma, A. Moon, N. Ohigashi, T. Minamiguchi, Y. Tsunawaki. Nucl. Instr. Meth. Phys. Res. A., 358, 399 (1995).

[20] M. Asakawa, N. Nakao, H. Ohkubob, T. Ishida, T. Watanabec, E. Yasuda, Y. Okudad, M. Fujita, J. Chen, A. Moon, P.K. Royb, S. Kuruma, K. Imasaki, K. Mimah, N. Ohigashi, Y. Tsunawakid, S. Nakaib, C. Yamanakan. Nucl. Instr. Meth. Phys. Res. A, 375, 416 (1996).

[21] D. Iracane, P. Bamas. Phys. Rev. Lett., 67, 3086 (1991).

[22] K. Lee, J. Mun, S.H. Park, K.-H. Jang, Y.U. Jeong, N.A. Vinokurov. Nucl. Instrum. Meth. Phys. Res. A, 776, 27 (2015).

[23] K. Halbach. J. Phys. Colloques, 44, C1 (1983).

[24] K. Halbach. J. Appl. Phys., 57 (8), 3605 (1985).

[25] B. Prakash, V. Huse, M. Gehlot, G. Mishra, S. Mishra. Optik, 127, 1639 (2016).

[26] V. Huse, G. Sharma, S. Mishra, G. Mishra. Chin. Phys. Lett., 31 (3), 034101 (2014).

[27] H. Jeevakhan, G. Mishra. Nucl. Instr. Meth. Phys. Res. A, 656, 101 (2011).

[28] V. Gupta, G. Mishra. Nucl. Instr. Meth. Phys. Res. A, 574, 150 (2007).

[29] V. Gupta, G. Mishra. Nucl. Instr. Meth. Phys. Res. A, 556 (1), 350 (2006).

[30] Y. Yang, W. Ding. Physics of Plasmas, 5 (3), 782 (1998).

[31] G. Mishra, A. Sharma. Nucl. Instr. Meth. Phys. Res. A, 976, 164287 (2020).

[32] Th. Schmidt, A. Anghel, P. Böhler, M. Brügger, M. Calvi, S. Danner, P. Huber, A. Keller, M. Locher. Magnetic Design of an APPLE III Undulator for SWISSFEL, Proc. FEL2014 (Basel, Switzerland, 2014). 116 MOP043 (http://accelconf.web.cern.ch/AccelConf/FEL2014/ papers/mop043.pdf).
[33] A.B. Temnykh. Phys. Rev. ST Accel. Beams, 11, 120702 (2008).

[34] H.-D. Nuhn, S.D. Anderson, R.N. Coffee, Y. Ding, Z. Huang, M. Ilchen, Yu.I. Levashov, A.A. Lutman, J.P. MacArthur, A. Marinelli, S.P. Moeller, F. Peters, Z.R. Wolf, J. Buck, G. Hartmann, J. Viefhaus, A.O. Lindahl, A.B. Temnykh. Commissioning of the Delta Polarizing Undulator at LCLS, Proc. FEL2015 (Daejeon, Korea, 2015). 757WED01.

[35] J.R. Henderson, L.T. Campbell, H.P. Freund, B.W.J. McNeil. New J. Phys., 18, 062003 (2016).

[36] K. Zhukovsky. J. Electromagn. Waves Appl., 28 (15), 1869 (2014).

[37] К.В. Жуковский. УФН, 2020. DOI: $10.3367 /$ UFNr.2020.06.038803.

[38] K. Zhukovsky. Opt. Laser Technology, 131, 106311 (2020).

[39] H. Jeevakhan, G. Mishra. Opt.Comm., 335, 126 (2015).

[40] K.V. Zhukovsky. J. Math. Anal. Appl., 446, 628 (2017).

[41] H.-S. Kang, C.-K. Min, H. Heo, C. Kim, H. Yang, G. Kim, I. Nam, S.Y. Baek, H.-J. Choi, G. Mun, B.R. Park, Y.J. Suh, D.C. Shin, J. Hu, J. Hong, S. Jung, S.-H. Kim, K.H. Kim, D. Na, S.S. Park, Y.J. Park, J.-H. Han, Y.G. Jung, S.H. Jeong, H.G. Lee, S. Lee, S. Lee, W.-W. Lee, B. Oh, H.S. Suh, Y.W. Parc, S.-J. Park, M.H. Kim, N.-S. Jung, Y.-C. Kim, M.S. Lee, B.-H. Lee, C.-W. Sung, I.-S. Mok, J.-M. Yang, C.-S. Lee, H. Shin, J.H. Kim, Y. Kim, J.H. Lee, S.-Y. Park, J. Kim, J. Park, I. Eom, S. Rah, S. Kim, K.H. Nam, J. Park, J. Park, S. Kim, S. Kwon, S.H. Park, K.S. Kim, H. Hyun, S.N. Kim, S. Kim, S.-M. Hwang, M. J. Kim, C.-Y. Lim, C.-J. Yu, B.-S. Kim, T.H. Kang, K.-W. Kim, S.-H. Kim, H.-S. Lee, H.-S. Lee, K.H. Park, T.-Y. Koo, D.-E. Kim, I.S. Ko. Nature Photonics, 11, 708 (2017).

[42] P. Emma. First lasing of the LCLS X-ray FEL at $1.5 \AA$, TH3PBI01, Proceedings of PAC09, Vancouver, BC, Canada, (2009)

[43] P. Emma, R. Akre, J. Arthur, R. Bionta, C. Bostedt, J. Bozek, A. Brachmann, P. Bucksbaum, R. Coffee, F.J. Decker, Y. Ding, D. Dowell, S. Edstrom, A. Fisher, J. Frisch, S. Gilevich, J. Hastings, G. Hays, P. Hering, Z. Huang, R. Iverson, H. Loos, M. Messerschmidt, A. Miahnahri, S. Moeller, H.D. Nuhn, G. Pile, D. Ratner, J. Rzepiela, D. Schultz, T. Smith, P. Stefan, H. Tompkins, J. Turner, J. Welch, W. White, J. Wu, G. Yocky, J. Galayda. Nature, Photonics, 4, 641 (2010).

[44] Н.А. Винокуров, Е.Б. Левичев. УФН, 185, 917 (2015).

[45] G. Geloni, E. Saldin, E. Schneidmiller, M. Yukov. Opt. Comm. 271, 207 (2007).

[46] Д.Ф. Алферов, Ю.А. Башмаков, П.А. Черенков. УФН, 157 (3), 389 (1989).

[47] В.Г. Багров, Г.С. Бисноватый-Коган, В.А. Бордовицын. Теория излучения релятивистских частиц(Физматлит, M., 2002)

[48] В.Г. Багров, И.М. Тернов, Б.В. Холомай. Излучение релятивистских электронов в продольном периодическом электрическом поле кристалла (ТФ СО АН СССР, Томск, 1987)

[49] Д.Ф. Алферов, Ю.А. Башмаков, Е.Г. Бессонов. ЖТФ, 43 (10), 2126 (1973).

[50] Е.Г. Бессонов. К теории источников ондуляторного излучения, Препринт ФИАН № 18, 1982.

[51] D. Ratner, A. Brachmann, F.J. Decker, Y. Ding, D. Dowell, P. Emma, A. Fisher, J. Frisch, S. Gilevich, Z. Huang, P. Hering, R. Iverson, J. Krzywinski, H. Loos, M. Messerschmidt, H.D. Nuhn, T. Smith, J. Turner, J. Welch, W. White, J. Wu. Phys. Rev ST-AB, 14, 060701 (2011). 
[52] S. Owada, K. Togawa, T. Inagaki, T. Hara, T. Tanaka, Y. Joti, T. Koyama, K. Nakajima, H. Ohashi, Y. Senba, T. Togashi, K. Tono, M. Yamaga, H. Yumoto, M. Yabashi, H. Tanaka, T. Ishikawa. J. Synchrotron Rad., 25, 282 (2018).

[53] Ichiro Inoue. Nat. Photon., 13, 319 (2019).

[54] http://xfel.riken.jp/eng/users/bml02-11.html

[55] H. Ego. RF system of the SPring-8 upgrade project, Proceedings of IPAC2016, Busan, Korea, MOPMW009.

[56] K. Tono, T. Hara, M. Yabashi, H. Tanaka. J. Synchrotron Rad., 26, 595 (2019)

[57] T. Ishikawa, H. Aoyagi, T. Asaka, Y. Asano1, N. Azumi, T. Bizen, H. Ego, K. Fukami, T. Fukui, Y. Furukawa, S. Goto, H. Hanaki, T. Hara, T. Hasegawa, T. Hatsui, A. Higashiya, T. Hirono, N. Hosoda, M. Ishii, T. Inagaki, Y. Inubushi, T. Itoga, Y. Joti, M. Kago, T. Kameshima, H. Kimura, Y. Kirihara, A. Kiyomichi, T. Kobayashi, C. Kondo, T. Kudo, H. Maesaka, X.M. Maréchal, T. Masuda, S. Matsubara, T. Matsumoto, T. Matsushita, S. Matsui, M. Nagasono, N. Nariyama, H. Ohashi, T. Ohata, T. Ohshima, S. Ono, Y. Otake, C. Saji, T. Sakurai, T. Sato, K. Sawada, T. Seike, K. Shirasawa, T. Sugimoto, S. Suzuki, S. Takahashi, H. Takebe, K. Takeshita, K. Tamasaku, H. Tanaka, R. Tanaka, T. Tanaka, T. Togashi, K. Togawa, A. Tokuhisa, H. Tomizawa, K. Tono, S. Wu, M. Yabashi, M. Yamaga, A. Yamashita, K. Yanagida, C. Zhang, T. Shintake, H. Kitamura, N. Kumagai. Nat. Photon., 6, 540 (2012).

[58] K. Tono, T. Togashi, Y. Inubushi, T. Sato, T. Katayama, K. Ogawa, H. Ohashi, H. Kimura, S. Takahashi, K. Takeshita, H. Tomizawa, S. Goto, T. Ishikawa, M. Yabashi. New J. Phys., 15, 083035 (2013).

[59] C.J. Milne, T. Schietinger, M. Aiba, A. Alarcon, J. Alex, A. Anghel, V. Arsov, C. Beard, P. Beaud, S. Bettoni, M. Bopp,

H. Brands, M. Brönnimann, I. Brunnenkant, M. Calvi, A. Citterio, P. Craievich, M.C. Divall, M. Dällenbach, M. D’Amico, A. Dax, Y. Deng, A. Dietrich, R. Dinapoli, E. Divall, S. Dordevic, S. Ebner, C. Erny, H. Fitze, U. Flechsig, R. Follath, F. Frei, F. Gärtner, R. Ganter, T. Garvey, Z. Geng, I. Gorgisyan, C. Gough, A. Hauff, C.P. Hauri, N. Hiller, T. Humar, S. Hunziker, G. Ingold, R. Ischebeck, M. Janousch, P. Juranić, M. Jurcevic, M. Kaiser, B. Kalantari, R. Kalt, B. Keil, C. Kittel, G. Knopp, W. Koprek, H.T. Lemke, T. Lippuner, D.L. Sancho, F. Löhl, C. Lopez-Cuenca, F. Märki, F. Marcellini, G. Marinkovic, I. Martiel, R. Menzel, A. Mozzanica, K. Nass, G.L. Orlandi, C.O. Loch, E. Panepucci, M. Paraliev, B. Patterson, B. Pedrini, M. Pedrozzi, P. Pollet, C. Pradervand, E. Prat, P. Radi, J.Y. Raguin, S. Redford, J. Rehanek, J. Réhault, S. Reiche, M. Ringele, J. Rittmann, L. Rivkin, A. Romann, M. Ruat, C. Ruder, L. Sala, L. Schebacher, T. Schilcher, V. Schlott, T. Schmidt, B. Schmitt, X. Shi, M. Stadler, L. Stingelin, W. Sturzenegger, J. Szlachetko, D. Thattil, D.M. Treyer, A. Trisorio, W. Tron 1, S. Vetter, C. Vicario, D. Voulot, M. Wang, T. Zamofing, C. Zellweger, R. Zennaro, E. Zimoch, R. Abela, L. Patthey, H.-H. Braun. Appl. Sci., 7, 720 (2017).

[60] R. Abela, P. Beaud, J.A. van Bokhoven, M. Chergui, T. Feurer, J. Haase, G. Ingold, S.L. Johnson, G. Knopp, H. Lemke, C.J. Milne, B. Pedrini, P. Radi, G. Schertler, J. Standfuss, U. Staub, L. Patthey. Struct. Dyn., 4, 061602 (2017).

[61] P. Juranić, J. Rehanek, C.A. Arrell, C. Pradervand, A. Cassar, M. Calvi, R. Ischebeck, C. Erny, P. Heimgartner, I. Gorgisyan, V. Thominet, K. Tiedtke, A. Sorokin, R. Follath, M. Makita, G. Seniutinas, C. David, C.J. Milne, H. Lemke, M. Radovic, C.P. Hauri, L. Patthey. J. Synchrotron Rad., 26, 906 (2019).
[62] R. Abela, A. Alarcon, J. Alex, C. Arrell, V. Arsov, S. Bettoni, M. Bopp, C. Bostedt, H.-H. Braun, M. Calvi, T. Celcer, P. Craievich, A. Dax, P. Dijkstal, S. Dordevic, E. Ferrari, U. Flechsig, R. Follath, F. Frei, N. Gaiffi, Z. Geng, C. Gough, N. Hiller, S. Hunziker, M. Huppert, R. Ischebeck, H. Jöhri, P. Juranic, R. Kalt, M. Kaiser, B. Keil, C. Kittel, R. Künzi, T. Lippuner, F. Löhl, F. Marcellini, G. Marinkovic, C. Ozkan Loch, G.L. Orlandi, B. Patterson, C. Pradervand, M. Paraliev, M. Pedrozzi, E. Prat, P. Ranitovic, S. Reiche, C. Rosenberg, S. Sanfilippo, T. Schietinger, T. Schmidt, K. Schnorr, C. Svetina, A. Trisorio, C. Vicario, D. Voulot, U. Wagner, H.J. Wörner, A. Zandonella, L. Patthey, R. Ganter. J. Synchrotron Rad., 26, 1073 (2019).

[63] S.V. Milton1, E. Gluskin, N.D. Arnold, C. Benson, W. Berg, S.G. Biedron, M. Borland, Y.-C. Chae, R.J. Dejus, P.K. Den Hartog, B. Deriy, M. Erdmann, Y.I. Eidelman, M.W. Hahne, Z. Huang, K.-J. Kim, J.W. Lewellen, Y. Li, A.H. Lumpkin, O. Makarov, E.R. Moog, A. Nassiri, V. Sajaev, R. Soliday, B.J. Tieman, E.M. Trakhtenberg, G. Travish, I.B. Vasserman, N.A. Vinokurov, X.J. Wang, G. Wiemerslage, B.X. Yang. Science, 292 (5524) 2037 (2001).

[64] S.G. Biedron, R.J. Dejus, Z. Huang, S.V. Milton, V. Sajaev, W. Berg, M. Borland, P.K. Den Hartog, M. Erdmann, W.M. Fawley, H.P. Freund,E. Gluskin, K.J. Kim, J.W. Lewellen, Y. Li, A.H. Lumpkin, E.R. Moog, A. Nassiri, G. Wiemerslage, B.X. Yang. Nucl. Instrum. Meth. Phys. Res. A, 483, 94 (2002).

[65] T. Tschentscher, C. Bressler, J. Grünert, A. Madsen, A.P. Mancuso. Appl. Sci., 7 (6), 592 (2017). 